\title{
Ultrastructural imaging reveals vascular remodeling in migraine patients
}

\author{
Katia Cortese ${ }^{1}$ (1) Erica Tagliatti ${ }^{2,3} \cdot$ Maria Cristina Gagliani $^{1} \cdot$ Matteo Frascio $^{4} \cdot$ Daniela Zarcone $^{1}$. \\ Edoardo Raposio ${ }^{4,5}$
}

Accepted: 9 December 2021

(c) The Author(s), under exclusive licence to Springer-Verlag GmbH Germany, part of Springer Nature 2021

\begin{abstract}
Migraine is a neurological disorder and one of the most common pain conditions worldwide. Despite its prevalence, the basic biology and underlying mechanisms contributing to the development of migraine are still poorly understood. It is still unclear, for instance, whether the vasculature, both extra and intracranial, plays a significant role in the generation of migraine pain. Neuroimaging data, indeed, have reported conflicting results on blood vessels abnormalities like vasodilation, while functional studies suggest that vessels dysfunction may extend beyond vasodilation. Here we combined light and electron microscopy imaging to investigate the fine structure of superficial temporal (STA) and occipital arteries (OA) from patients that underwent minimally invasive surgery for migraine. Using optical microscopy, we observed that both STA and OA vessels showed marked endothelial thickening and internal elastic lamina fragmentation. In the muscular layer, we found profound shape changes of vascular smooth muscle cells (VSMCs), abundant extracellular matrix, and the presence of clear extracellular vacuoles. The electron microscopy analysis confirmed putative VSMCs infiltrated within the intima layer and revealed a consistent shifting of VSMCs from contractile to a synthetically active phenotype. We also report the presence of (i) abundant extracellular vacuoles filled with fine granular material and membranes, (ii) multilamellar structures, (iii) endosome-like organelles, and (iv) bona fide extracellular vesicles in the matrix space surrounding synthetically active cells. As both the endothelial layer and VSMCs coordinate a variety of vascular functions, these results suggest that a significant vascular remodeling is occurring in STA and OA of migraine patients. Thus, this phenomenon may represent an important target for future investigation designed toward the development of new therapeutic approaches.
\end{abstract}

Keywords Migraine disorders $\cdot$ Pain $\cdot$ Phenotypic switching $\cdot$ Vascular remodeling $\cdot$ Microscopy $\cdot$ Vascular smooth muscle cell

Katia Cortese and Erica Tagliatti have contributed equally.

Katia Cortese

cortesek@unige.it

1 Cellular Electron Microscopy Laboratory, Department of Experimental Medicine (DIMES), Human Anatomy, University of Genova, Genoa, Italy

2 Laboratory of Pharmacology and Brain Pathology, Humanitas Clinical and Research Center, Rozzano, Milan, Italy

3 Department of Clinical and Experimental Epilepsy, UCL Queen Square Institute of Neurology, University College London, London, UK

4 Plastic Surgery Unit, Department of Surgical Sciences and Integrated Diagnostics (DISC), University of Genova, Genoa, Italy

5 Plastic and Reconstructive Surgery Division, San Martino University Hospital, Genova, Italy

\section{Introduction}

Migraine is a complex neurological disorder with a strong genetic component and is one of the most common pain conditions worldwide (Ashina et al. 2021; Eigenbrodt et al. 2021). There are rare monogenic forms of migraine, as well as more common polygenic forms. Research into the genes involved in both types has provided insights into the role of both neural and vascular pathways (Sutherland et al. 2019). Numerous studies have also indicated that migraineurs have an increased risk of vascular diseases (Cavestro et al. 2021). For example, an association between migraine and ischemic stroke was earliest recognized (Øie et al. 2020). Other studies regarding arterial function in migraine pathogenesis have shown a greater stiffness or impaired compliance of the arterial system in migraine patients (Ripa et al. 2015). However, 
the role played by the vasculature in migraine pathogenesis still remains controversial (Sacco et al. 2013; Mason and Russo 2018). The widely accepted vascular theory, indeed, based on the vasodilation of cerebral and meningeal arterial as the primary migraine trigger, has been recently refuted by a growing body of research.

Amin et al. (2013), for example, based on their negative correlation between migraine pain dilation of extracranial arteries, concluded that vasodilation does not primarily cause migraine symptoms.

While vasodilation itself may not contribute to migraine, it remains possible that vessels play a role in migraine even in the absence of vasodilation. Therefore, it appears that mechanisms underlying the association between migraine and altered arterial function are likely to be more complex and may involve both structural and functional changes in the arterial wall.

Blood vessels are dynamic structures constantly subjected to intense remodeling under different conditions. At the microscopic level, arteries and arterioles consist of an endothelium and basement membrane lining the inner vessel wall (tunica intima), a smooth muscle layer (tunica media), which mediates contraction and dilation, and a connective tissue layer (tunica adventitia) in contact with the surrounding tissue. The artery is innervated in two main layers: within the tunica media (myoneural synapses), and in the adventitial layer (sensory nerve endings).

Vascular smooth muscle cells (VSMCs) in the tunica media are essential for good performance of the vasculature (Rensen et al. 2007; Lacolley et al. 2012).

They regulate vascular tone primarily in response to sympathetic nervous system innervation (e.g., adrenergic receptor activation), nitric oxide (NO), as well as the local synthesis, uptake, and release of 5-HT. Many vascular diseases show microscopic abnormalities in this cell layer (Eid et al. 2012; Kapustin and Shanahan 2016; Petsophonsakul et al. 2019). For example, the shift of smooth muscle cell phenotype from contractile to synthetically active is one of the first signs of vascular injury. Under pathological conditions, VSMCs can dedifferentiate into a synthetically active phenotype, starting secretion of extracellular vesicles, cell proliferation, and migration to repair injury (Petsophonsakul et al. 2019). At the molecular level, this switching is regulated by different mechanisms, including autophagic activation (Salabei and Hill 2015). In addition to smooth muscle cells, endothelial cells might be strongly involved as well in the pathophysiology of migraine. These cells directly contact the circulating blood and also control vessel function. Endothelial cells are involved in the regulation of cell-cell barrier maintenance and, among others, vascular tone, vascular remodeling, immune surveillance, blood coagulation, and nutrient uptake. It is therefore clear that arteries do not simply provide a conduit for the movement of blood that can constrict and dilate, but rather they coordinate a much more intricate signaling between multiple cell types and in a bidirectional way with the nervous system. Thus, we could argue that dysregulation of any part of this specific vascular architecture and signaling process may contribute to migraine pathology (Jacobs and Dussor 2016).

Migraine surgery has been widely accepted as an effective surgical solution for chronic headaches refractory to medical and conservative treatment (Omranifard et al. 2016; Gfrerer et al. 2018). Four main trigger zones of migraine are amenable to surgical decompression: frontal (site I: supraorbital and supratrochlear nerves), temporal (site II: zygomatictemporal branch of the trigeminal nerve), endonasal (site III: trigeminal end branches), and occipital (site IV: great occipital nerve) (Bertozzi et al. 2018).

Modern neuroimaging techniques, although very sensitive and informative, do not provide sufficient resolution to detect microscopic vessel wall alterations. Here, we combined light and electron microscopy imaging on fresh biopsies of the superficial temporal artery (STA) and occipital artery (OA) obtained from a cohort of patients that underwent minimally invasive surgery (MIS) to investigate vessel alterations and provide new evidence in support of the theory of a vasculature role in migraine pathology.

\section{Materials and methods}

The study was conducted in compliance with the Declaration of Helsinki and the Guidelines for Good Clinical Practice; all patients provided written informed consent. Fifteen patients (six male, nine female, mean age 43.5 years, range 37-50 years) were subjected to MIS for migraine (Gfrerer et al. 2018). Fifteen biopsies, ten of the STAs and five from OAs, were collected under the surgical procedure and immediately processed for optical and ultramicroscopic analysis.

\section{Light and electron microscopy}

All samples were fixed in $2.5 \%$ glutaraldehyde in $0.1 \mathrm{M}$ cacodylate buffer, postfixed in $1 \%$ osmium tetroxide, stained en bloc with $1 \%$ aqueous uranyl acetate, and dehydrated in graded alcohols and propylene oxide. Then samples were embedded in an Epoxy resin mixture and polymerized for 2 days at $60{ }^{\circ} \mathrm{C}$. For light microscopy, $200 \mathrm{~nm}$ semithin sections were cut until the complete three layers of the vessel were detected and stained with $1 \%$ toluidine blue. Fiftynanometer ultrathin sections were counterstained with 5\% uranyl acetate in 50\% ethanol and lead citrate. Electron micrographs from two levels of sectioning were acquired with a Hitachi HT77800 $120 \mathrm{kV}$ electron microscope (Hitachi, Tokyo, Japan) operating at $100 \mathrm{kV}$, equipped with a Megaview G3 digital camera and Radius software (EMSIS, 
Muenster, Germany, version 2.0) using the snapshot and the multiple image alignment (MIA) montage tools.

\section{Results and discussion}

\section{Light microscopy of STA and OA}

Ten STA and five OA fresh biopsies from patients affected by migraine (median age of 46) were collected during a MIS procedure and immediately processed for light and electron microscopy analysis. In normal muscular arteries, the tunica intima (endothelial layer) consists of a single layer of endothelial cells surrounded by a connective tissue basement membrane with elastic fibers. The middle layer, the tunica media, is primarily composed of smooth muscle and is usually the thickest layer. We first analyzed $200 \mathrm{~nm}$ semithin sections stained with $1 \%$ toluidine blue of all STA and OA samples. Toluidine blue (TB) is a basic thiazine metachromatic dye with high affinity for acidic tissue components, thereby staining tissues rich in DNA and RNA. In addition, being a cationic dye, TB staining visualizes well proteoglycans in the extracellular matrix (Sridharan and Shankar 2012). Alterations in the morphological structure of the vessel wall were observed in all STA and OA samples analyzed. The endothelial layer forms a continuous sheet of flattened and slightly polygonal cells around the lumen of the vessel, which appear narrowed and filled with red blood cells (RBCs) (Fig. 1a, b, e, f). Interestingly, we report intimal hyperplasia (Fig. 1a-c), fragmentation of the internal elastic lamina (IEL) with infiltration of polygonal cells with pale nuclei (Figs. 1c, d, 2b, 4a). Intimal hyperplasia was variable in its extent but present in all samples analyzed, as well as fragmentation of the IEL. These data are suggestive of an active process of vascular remodeling, which has already been reported occurring in distinct vascular pathologies (Renna et al. 2013; Kapustin and Shanahan 2016). The tunica media or muscular layer normally consists of a thick layer of concentrically arranged smooth muscle fibers. In STA and OA biopsies, we observed marked irregularity in
Fig. 1 Light microscopy of toluidine blue-stained $500 \mathrm{~nm}$ semithin sections from a representative superficial temporal artery biopsy from a female patient (\#1). a Low-magnification toluidine blue-stained STA (magnification $\times 10$ ). $\mathbf{b}$ Same image as in (a) with the three vessel layers depicted with different colors (magnification $\times 10$ ), internal elastic lamina (IEL). $\mathbf{c}$ Intimal to tunica media border with internal elastic lamina (IEL) showing marked thickening (magnification $\times 20$ ). d Inset box of image (c) showing internal elastic lamina (IEL) fragmentation (black arrowheads) and infiltrating polygonal cells (black arrows), vascular smooth muscle cells (VSMCs) magnification $\times 40$. e Lumen of the vessel filled with erythrocytes (BRCs) and endothelial lining (magnification $\times 20$ ). $\mathbf{f}$ Inset box of the image in (e) showing endothelial cells (EC, black arrowheads) and several polygonal cells with pale cytoplasm and heterochromatin poor nuclei underneath the endothelial layer (black arrows) (magnification $\times 40)$, red blood cells (RBCs). Scale bars: $200 \mu \mathrm{m}$ (a, b), $50 \mu \mathrm{m}$ (c, e), $20 \mu \mathrm{m}(\mathbf{d}, \mathbf{f})$
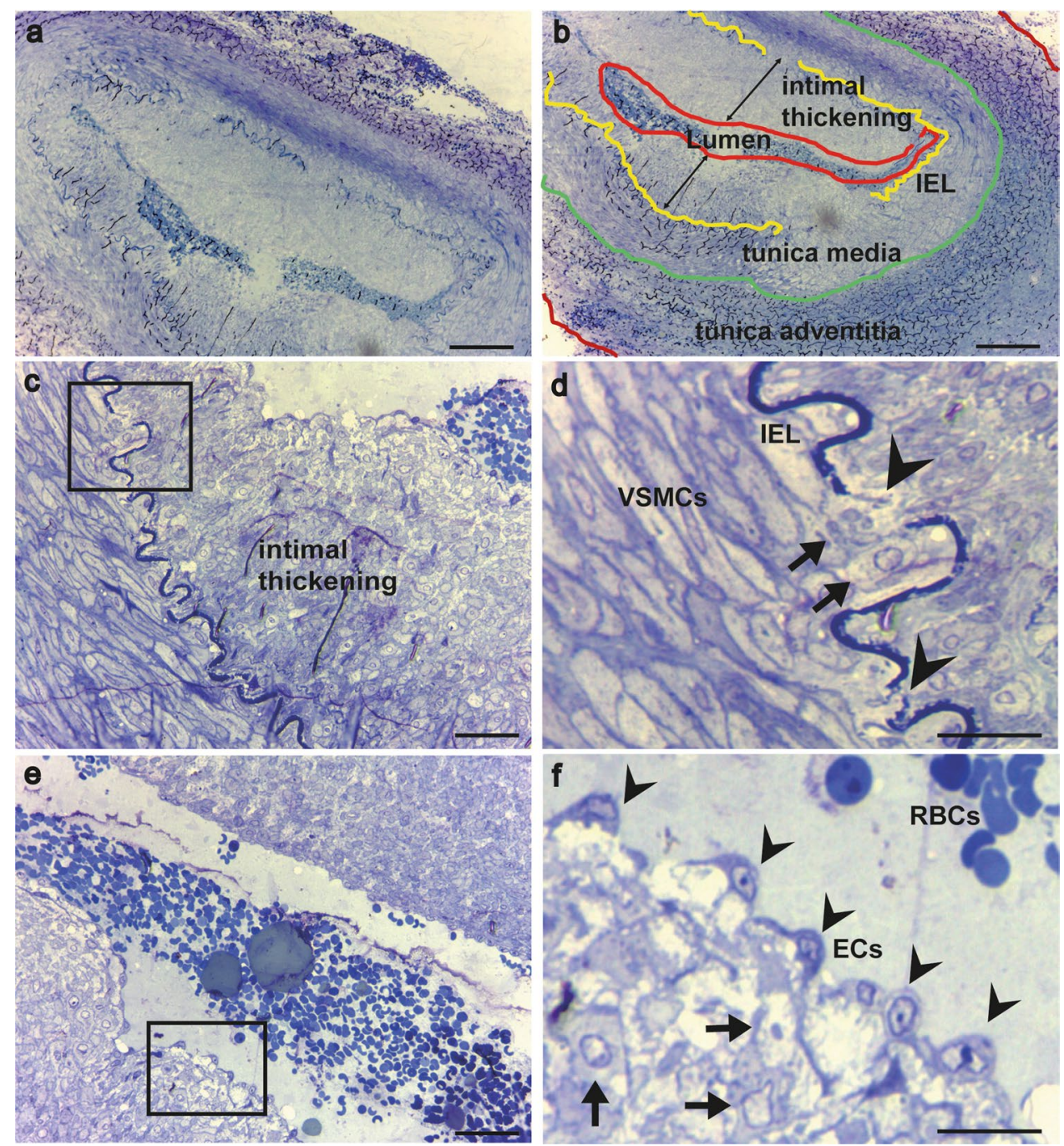

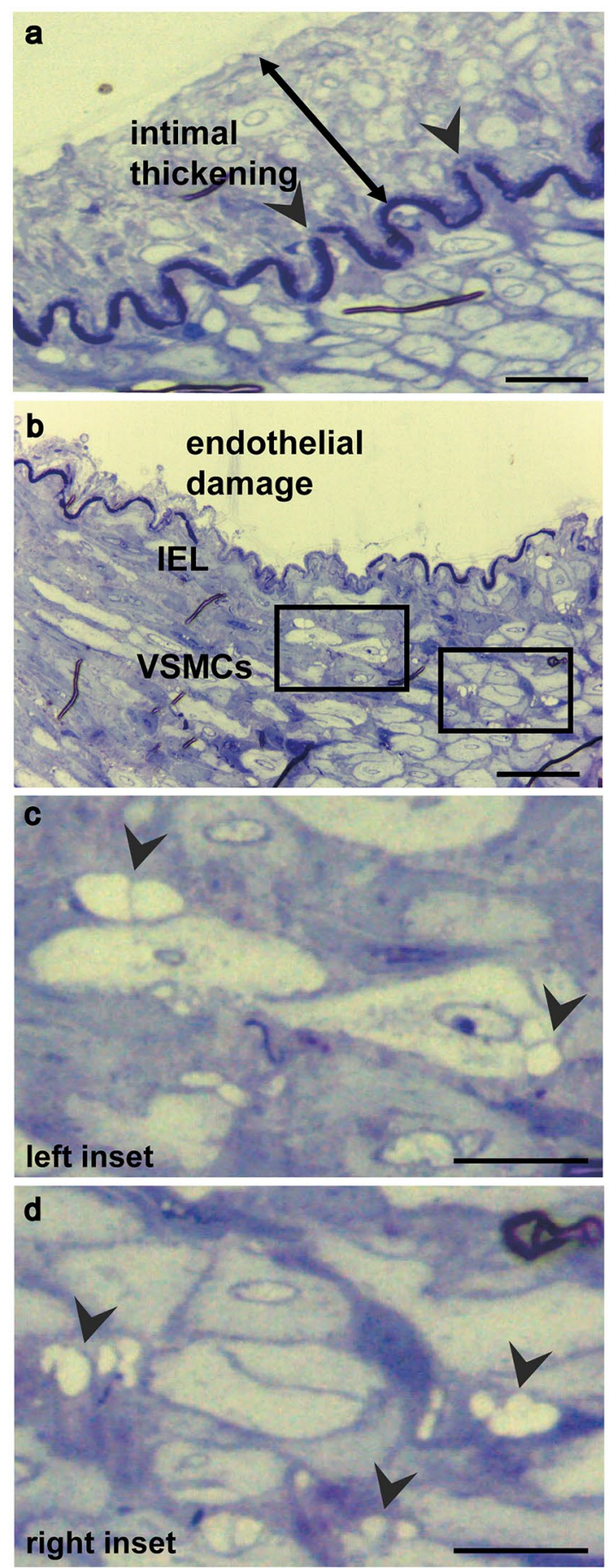

Fig. 2 Light microscopy of toluidine blue stained $200 \mathrm{~nm}$ semithin sections from two representative superficial temporal artery biopsies from two female patients (\#2, \#3). Representative images showing endothelial damage with IEL fragmentation (a), intimal hyperplasia (b), and limited metachromasia of VSMCs of different shapes (a, b). In (b) are also depicted two inset boxes enlarged in (c, d). Inset boxes from (b) showing VSMCs in the muscular layer surrounded by clear vacuoles (black arrowheads) and extracellular matrix (c, d). Scale bar: $50 \mu \mathrm{m}(\mathbf{a}, \mathbf{b}), 20 \mu \mathrm{m}(\mathbf{c}, \mathbf{d})$ the shape of VSMCs, different TB metachromasia of cells, separation of cells by abundant TB-stained extracellular matrix and clear vacuoles of different size (Fig. 2a, b). These results suggested a possible phenotype switching occurring in the VSMC layer (Petsophonsakul et al. 2019), consistent with vascular remodeling.

\section{Ultrastructural changes in STA and OA}

To investigate whether the cells in tunica media were effectively undergoing a phenotype switching, we performed ultrastructural analysis on $50 \mathrm{~nm}$ ultrathin sections of STA and OA samples. VSMCs are highly specialized cells that regulate vascular tone and participate in vessel remodeling in physiological and pathological conditions (Lacolley et al. 2012). It has been reported that VSMC phenotypic conversion from a contractile and quiescent to synthetically active state contributes to vascular pathologies including restenosis, atherosclerosis, and vascular calcification (Eid et al. 2012; Kapustin and Shanahan 2016; Pahk et al. 2017). In contrast to contractile cells, synthetically active cells proliferate, migrate, synthesize components of the extracellular matrix and actively release exosomes within the extracellular space (Kapustin and Shanahan 2016).

We observed, in both STA and OA samples, the presence of a heterogeneous population of VSMCs cells characterized by four distinct ultrastructural aspects. First, we show that the major fraction of synthetically active smooth muscle cells displayed a polygonal shape, irregular borders with migratory appearance, less packed myofilaments in the cytoplasm, reduced dense bodies, presence of lipofuscin, and abundant extracellular matrix (Fig. 3a). This population of cells coexisted with cells in a transitional state (Fig. 3b) and, to a lesser extent, with fully contractile cells (Fig. 3c). Many putative synthetically active cells, showing the ultrastructural morphology of VSMCs, were found in the intimal layer, suggesting migration of these cells from the tunica media and contributing to the thickening observed (Fig. 4a, b). Second, in the muscular layer, the interstitial spaces were considerably widened and filled with increased amounts of collagen and extracellular matrix (ECM). Third, bona fide extracellular vesicles (EVs) and multilamellar structures, as well as small endosome-like organelles, were observed embedded in the context of ECM (Fig. 5a, b). Fourth, several membrane-bound vacuoles of different sizes and filled with fine granular material were found in the extracellular space among synthetically active VSMCs. Occasionally, we also detected vesicular profiles budding inside the vacuoles (Fig. 5c, inset) and internal membranes (Fig. 5d), strongly suggestive of their endocytic nature. Interestingly, these structures were reminiscent of ghost bodies observed in early studies and are described as an exocytotic process in which cytoplasmic material is discarded by the cell in the 

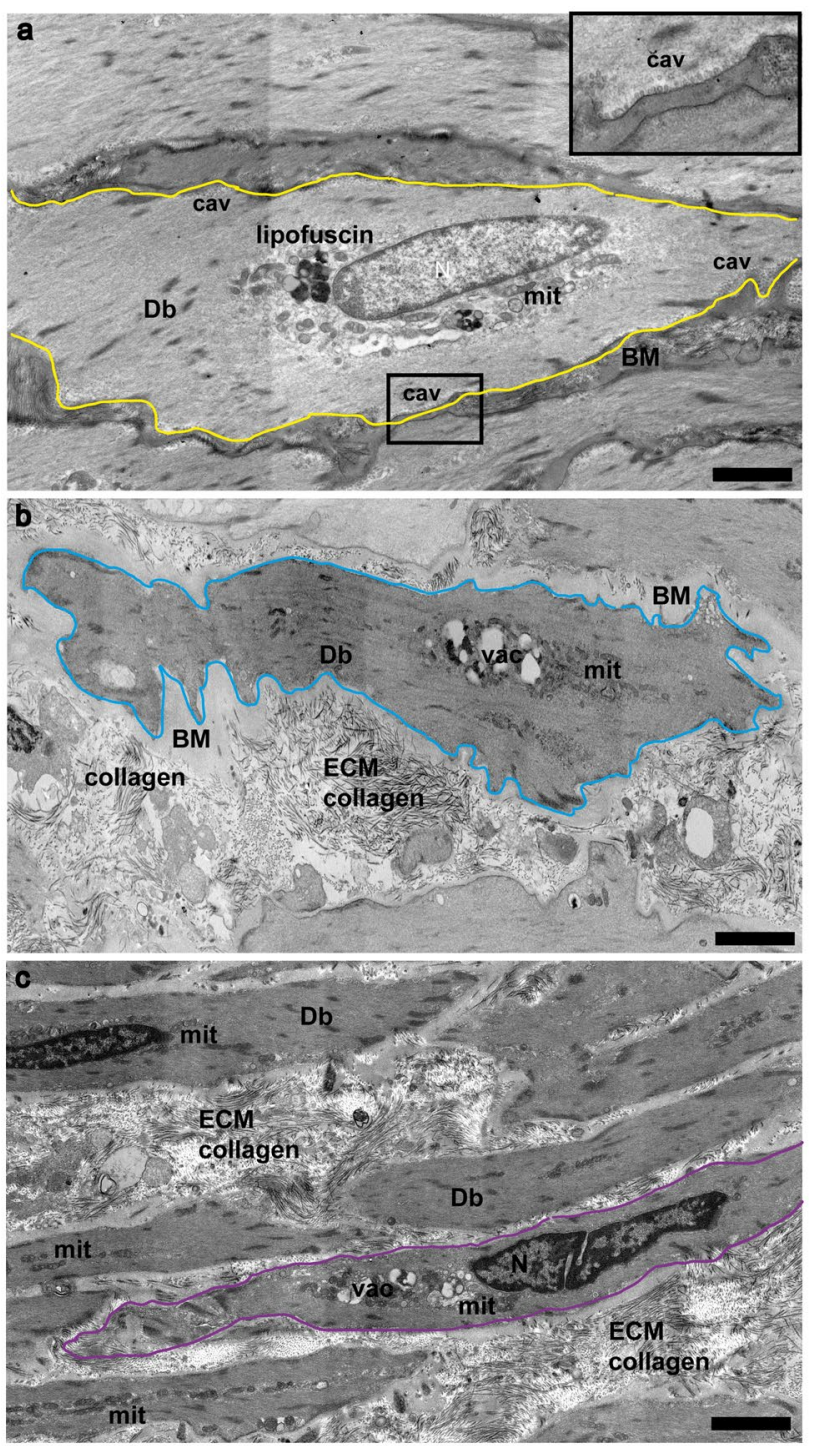

Fig. 3 Electron micrographs of the cellular phenotypes present in the muscular layer of occipital artery biopsies (patient \#1 and \#2). In (a) is shown a multiple image alignment (MIA) montage of a synthetically active cell (yellow borders) with clear cytoplasm, reduced filaments and dense bodies ( $\mathrm{Db})$, and presence of lipofuscin and mitochondria (mit) near the nuclear region. The inset shows the plasma membrane of the transitional cell decorated with abundant caveolae. In (b) is shown a MIA montage of smooth muscle cell with a transitional phenotype (border in light blue). Note the increased electron density compared to cells in (a), the irregular shape, presence of intracellular vacuoles (vac), mitochondria (mit), irregular basal membranes (BM), and of extracellular spaces filled with abundant collagen and extracellular matrix (ECM). MIA montage of smooth muscle cells with the contractile phenotype (border in violet) in (c). Note the fusiform shape, dense bodies (Db), nucleus $(\mathrm{N})$, mitochondria (mit), intracellular vacuoles (vac), and extracellular matrix (ECM), mainly collagen fibers in the surrounding space. Scale bar: $2 \mu \mathrm{m}(\mathbf{a}-\mathbf{c})$

extracellular space (Trillo 1981). The nature of the granular material found within the vacuoles is currently unknown but we might speculate that myofilaments and contractile
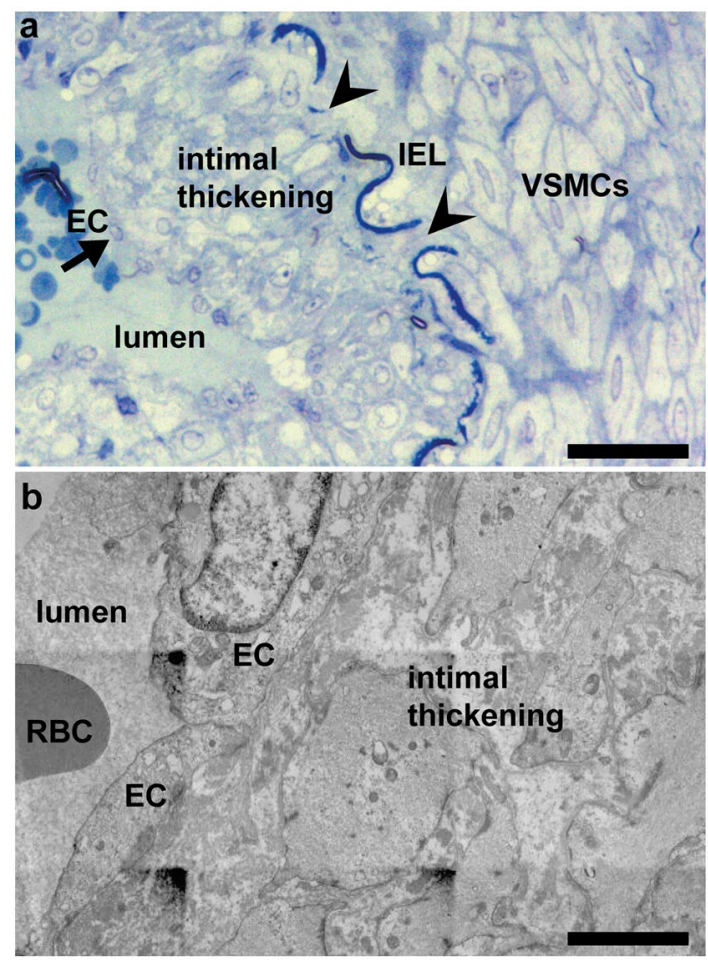

Fig. 4 Light and electron microscopy of intimal thickening of representative STA. In (a) is shown a toluidine blue stained $200 \mathrm{~nm}$ semithin section from a superficial temporal artery biopsy (female patient \#1) showing vessel lumen (lumen), a thickened endothelial layer comprising endothelial cells (EC, arrow) and polygonal cells with pale cytoplasm and heterochromatin-poor nuclei (intimal thickening), fragmented internal elastic lamina (IEL, arrowheads), and tunica media with VSMCs. In (b) are representative electron micrographs showing the lumen of the same vessel with a RBC, two adjacent endothelial cells (EC), and two putative VSMCs underneath ECs contributing to the intimal thickening. Scale bar: $30 \mu \mathrm{m}$ (a), scale bar: $2 \mu \mathrm{m}(\mathbf{b})$

machinery can be discarded from the cells as a result of phenotypical switching. Therefore, the presence of these extracellular vacuoles in all STA and OA samples analyzed might suggest that phenotypic switching is accompanied by a striking activation of degradative and/or autophagic processes (Fig. 5c, d).

Taken together, at the microscopic level, the modifications observed in the tunica intima and media of both STA and OA arteries support vascular injury and intense remodeling. Although these types of alterations cannot be considered specific for migraine as already described in different vascular disorders (e.g., hypertension and atherosclerosis) (Pahk et al. 2017), we should consider that in the history of our patients were not reported any of these latter pathologies. Therefore, we cannot exclude that vascular remodeling would represent a common early mechanism that is activated in different vascular pathologies. To our knowledge, this is the first report that describes vascular 

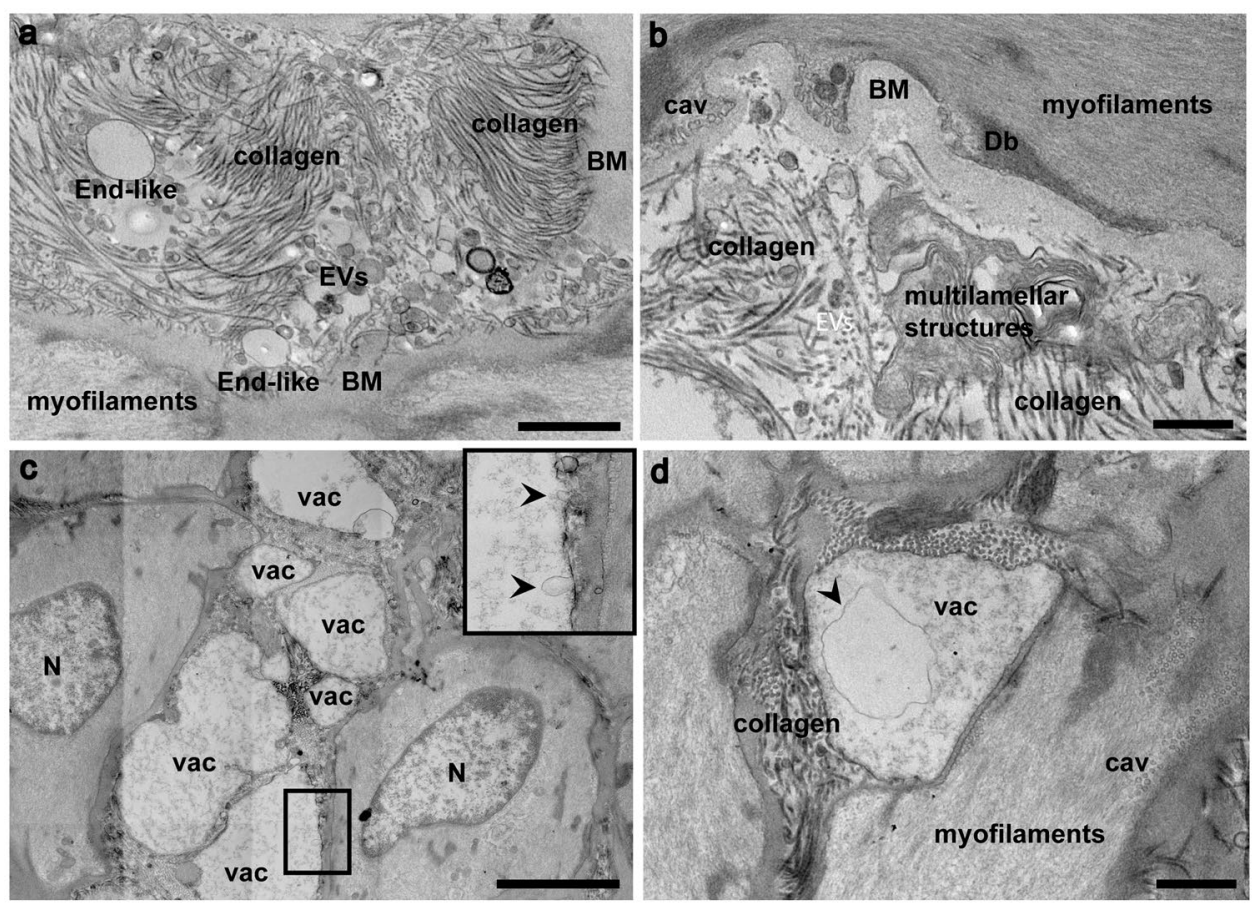

Fig. 5 Electron micrographs of extracellular matrix (ECM) among synthetically active cells of STA and OA biopsies. In (a) is depicted a representative image of a STA showing the basal membrane (BM) of a synthetically active cell, myofilaments, and extracellular matrix filled with collagen fibers (collagen), extracellular vesicles (EVs), and small endosome-like structures (endo-like). In (b) is shown a representative image of an OA showing the basal membrane of a synthetically active cell (BM), myofilaments, dense bodies (Db),

microscopic abnormalities in chronic migraine patients and supports the theory that vascular remodeling, as either direct or indirect effect, might play an important role in migraine. Further molecular studies will be needed for better understanding the underlying mechanisms in the development of the observed ultrastructural changes.

Acknowledgements We gratefully acknowledge the University of Genoa for funding the acquisition of the Hitachi HT7800 $120 \mathrm{kV}$ transmission electron microscope (Grant Heavy Equipment, D.R. 3404). We are also grateful to Chiara Fiocco for technical assistance.

Author contributions Study design: KC, ER. Study conduct: KC, ET, MCG, MF and DZ. Data collection: KC, ET, MCG, MF and DZ. Data analysis: KC, ET, MCG. Data interpretation: KC, ET and ER. Drafting and submission of manuscript: KC, ET and ER.

Funding Funded by Università degli Studi di Genova, Katia Cortese, (Grant No. 100008-2020-EC-FRA_001_FRA2020), (Grant No. D.R. 3404, Grant Heavy Equipment)

Data availability The datasets generated during and/or analyzed during the current study are available from the corresponding author on reasonable request. caveolae (Cav), multilamellar structures, collagen fibers, and extracellular vesicles (EVs). Membrane-bound vacuoles (vac) filled with fine granular material immersed in the extracellular space surrounding synthetically active cells from STA are shown in $(\mathbf{c}, \mathbf{d})$. Note in the inset in (c) the presence of vesicular profiles budding within the vacuole (arrowheads), as well as the internal membrane (arrowhead) indicated in (d), suggestive of a likely endocytic nature of these structures. Scale bar: $1 \mu \mathrm{m}$ (a), $500 \mathrm{~nm}(\mathbf{b}), 2 \mu \mathrm{m}$ (c), $500 \mathrm{~nm}(\mathbf{d})$

\section{Declarations}

Conflict of interest The authors have declared that no competing interests exist.

\section{References}

Amin FM, Asghar MS, Hougaard A et al (2013) Magnetic resonance angiography of intracranial and extracranial arteries in patients with spontaneous migraine without aura: a cross-sectional study. Lancet Neurol 12:454-461. https://doi.org/10.1016/S14744422(13)70067-X

Ashina M, Terwindt GM, Al-Karagholi MAM et al (2021) Migraine: disease characterisation, biomarkers, and precision medicine. Lancet 397:1496-1504. https://doi.org/10.1016/S0140-6736(20) 32162-0

Bertozzi N, Simonacci F, Lago GL et al (2018) Surgical therapy of temporal triggered migraine headache. Plast Reconstr Surg Glob Open 6:1-8. https://doi.org/10.1097/GOX.0000000000001980

Cavestro C, Degan D, Micca G et al (2021) Thrombophilic alterations, migraine, and vascular disease: results from a case-control study. Neurol Sci 42:3821-3828. https://doi.org/10.1007/ s10072-020-05006-z 
Eid RA, Radad K, Al-Shraim M (2012) Ultrastructural changes of smooth muscles in varicocele veins. Ultrastruct Path 36:201-206. https://doi.org/10.3109/01913123.2011.637663

Eigenbrodt AK, Ashina H, Khan S et al (2021) Diagnosis and management of migraine in ten steps. Nat Rev Neurol 17:501-514

Gfrerer L, Raposio E, Ortiz R, Gerald Austen W (2018) Surgical treatment of migraine headache: back to the future. Plast Reconstr Surg 142:1036-1045. https://doi.org/10.1097/PRS.0000000000004795

Jacobs B, Dussor G (2016) Neurovascular contributions to migraine: moving beyond vasodilation. Neuroscience 338:130-144. https:// doi.org/10.1016/j.neuroscience.2016.06.012

Kapustin AN, Shanahan CM (2016) Emerging roles for vascular smooth muscle cell exosomes in calcification and coagulation. $\mathrm{J}$ Physiol 594:2905-2914. https://doi.org/10.1113/JP271340

Lacolley P, Regnault V, Nicoletti A et al (2012) The vascular smooth muscle cell in arterial pathology: a cell that can take on multiple roles. Cardiovasc Res 95:194-204. https://doi.org/10.1093/cvr/ cvs 135

Mason BN, Russo AF (2018) Vascular contributions to migraine: Time to revisit? Front Cell Neurosci. https://doi.org/10.3389/FNCEL. 2018.00233

Øie LR, Øie LR, Kurth T et al (2020) Migraine and risk of stroke. J Neurol Neurosurg Psychiatry 91:593-604. https://doi.org/10. 1136/jnnp-2018-318254

Omranifard M, Abdali H, Ardakani M, Talebianfar M (2016) A comparison of outcome of medical and surgical treatment of migraine headache: In 1 year follow-up. Adv Biomed Res. https://doi.org/ 10.4103/2277-9175.186994

Pahk K, Joung C, Jung SM et al (2017) Visualization of synthetic vascular smooth muscle cells in atherosclerotic carotid rat arteries by F-18 FDG PET. Sci Rep 7:1-8. https://doi.org/10.1038/ s41598-017-07073-3

Petsophonsakul P, Furmanik M, Forsythe R et al (2019) Role of vascular smooth muscle cell phenotypic switching and calcification in aortic aneurysm formation involvement of Vitamin K-dependent processes. Arterioscler Thromb Vasc Biol 39:1351-1368. https:// doi.org/10.1161/ATVBAHA.119.312787

Renna NF, de Las Heras N, Miatello RM (2013) Pathophysiology of vascular remodeling in hypertension. Int J Hypertens. https://doi. org/10.1155/2013/808353

Rensen SSM, Doevendans PAFM, van Eys GJJM (2007) Regulation and characteristics of vascular smooth muscle cell phenotypic diversity. Neth Heart J 15:100-108. https://doi.org/10.1007/ BF03085963

Ripa P, Ornello R, Pistoia F et al (2015) Spreading depolarization may link migraine, stroke, and other cardiovascular disease. Headache 55:180-182. https://doi.org/10.1111/head.12436

Sacco S, Ripa P, Grassi D et al (2013) Peripheral vascular dysfunction in migraine: a review. J Headache Pain 14:80. https://doi.org/10. $1186 / 1129-2377-14-80$

Salabei JK, Hill BG (2015) Autophagic regulation of smooth muscle cell biology. Redox Biol 4:97-103. https://doi.org/10.1016/j. redox.2014.12.007

Sridharan G, Shankar AA (2012) Toluidine blue: a review of its chemistry and clinical utility. J Oral Maxillofac Pathol 16:251-255. https://doi.org/10.4103/0973-029X.99081

Sutherland HG, Albury CL, Griffiths LR (2019) Advances in genetics of migraine. J Headache Pain. https://doi.org/10.1186/ s10194-019-1017-9

Trillo AA (1981) Formation of "ghost" bodies and calcification in experimental atherosclerosis in nonhuman primates-An Ultrastructural Study. Virchows Arch 38:127-139. https://doi.org/10. 1007/BF02892808

Publisher's Note Springer Nature remains neutral with regard to jurisdictional claims in published maps and institutional affiliations. 